\title{
Analysis Regarding Tracking Phenomenon to Adult Obesity
}

Fujii $K^{*}$

Graduate School of Business Administration and Computer Science, Aichi Institute of Technology, Japan

${ }^{*}$ Corresponding author: Fujii K, Graduate School of Business Administration and Computer Science, Aichi Institute of Technology, Japan, E-mail: fujii@aitech.ac.jp

Citation: Fujii K (2018) Analysis Regarding Tracking Phenomenon to Adult Obesity. J Nutr Health Sci 5(3): 307. doi: $10.15744 / 2393-9060.5 .307$

Received Date: July 12, 2018 Accepted Date: September 12, 2018 Published Date: September 14, 2018

\begin{abstract}
The body transition in becoming adults of obese and lean types has not been clearly demonstrated. In this study we first investigated the changes in mean BMI with age by applying the wavelet interpolation method to the changes with age in girls' BMI. Then, by detecting the maximum peak velocity in the age-related changes in that BMI (MPV of BMI), we confirmed that BMI, similar to height and weight, follows a general type growth pattern tracing a sigmoid curve in which the peak appears in puberty. Author then constructed an obese-lean evaluation chart that incorporates the age-related changes in BMI, and conducted a longitudinal investigation of the rates of appearance of obesity and leanness. The normality of BMI levels in each year from the first to the ninth grades was then investigated and the wavelet interpolation method was applied to the reference values obtained by establishing 5 -step mean evaluation reference values. An obese evaluation chart was then constructed from the derived standard age-related change curve, and obese types were determined. The rate of appearance of these types from the first grade to the ninth grade was then investigated longitudinally. Author attempt to determine whether the BMI age-related change evaluation chart is valid in determinations of obesity.
\end{abstract}

Keywords: Obesity; Change of BMI with age; Tracking; Wavelet Interpolation Model

\section{Introduction}

In recent years body mass index (BMI) has been widely used in medicine internationally as an indicator of obesity, such as in the definition of obesity by the WHO. The obesity is defined as abnormal or excessive fat accumulation that presents a risk to health. A person with a BMI of 25 or more is considered by WHO to be overweight. BMI is derived from values for body height and weight [weight $(\mathrm{kg}) /$ height $(\mathrm{m})^{2}$ ], while in early childhood the Kaup index [body weight $(\mathrm{g}) /(\text { height }(\mathrm{cm}))^{2} \times 10$ ] is used to determine level of obesity in young children. BMI was originally an index of physique using body weight in proportion to the square of height, developed by Quetelet (1985) for French soldiers. A correlation with body fat percentage was verified by Key (1972) and since that time BMI has come to be widely taken up [1]. At the same time, however, BMI is also known to be deeply linked to sickness and health. For example, Lee et al. (1996) and Rikli and Jones (1998) reported that it is used in evaluation indices showing those possibilities [2,3]. A relationship between BMI and physical fitness has also been suggested, with Arao (1998)(1999), Coakly et al. (1998), Davis et al. (1998), and Launer et al. (1994) reporting lower physical fitness in people with higher BMI [4-8]. Ferraro and Booth (1999) and Galanos et al. (1994) also reported low physical fitness in people with either very high or very low BMI $[9,10]$. These studies were done with elderly subjects and the reports suggest that BMI is an unvarying state. To our knowledge, there are no findings on BMI and health or sickness in school-age children. This means that since BMI changes considerably with age in schoolchildren, no BMI indicators have been formed for this period. The Rohrer index has been applied to assess obesity in schoolchildren, behind which is the fact that the Rohrer index does not fluctuate that much during the school years. However, Takaishi and Omori (1978) attempted a longitudinal approach to the age trends in the Rohrer index, and investigated the pattern of the change with age. They showed a gentle concave line downward on average, and reported that after puberty there is a more dramatic rise in girls than in boys. Looking at individuals, they reported dramatic fluctuations and showed a pattern of nearly parallel trends with age in about half of subjects, and from these changes with age they questioned whether the Rohrer index was useful in the assessment of obesity. Rather, when BMI shows an increase with age, it would seem that if those increasing trends could be ascertained criteria could be constructed with them. However, for a stable expression of the level of obesity, BMI standard values must not fluctuate greatly in order for obesity to be assessed accurately. In other words, effectiveness is sought in making determinations with BMI only without consideration of age. Therefore, negative interpretations are dominant for application to school-age children, and there are not thought to be any established measures. In actual practice, the Kaup index is applied in 
early childhood and BMI is applied with adults, but it is still an index. Ultimately, this is nothing more than establishing different standard values for children and adults. If that is the case, it is thought that the range of use of BMI could be expanded if unique standard values could be constructed for the school-age period from early childhood to adulthood. For that purpose the changes with age in BMI need to be investigated. However, from a line graph with raw BMI data plotted by age it is difficult to precisely analyze the changes with age. Drawing a smooth percentile curve showing those standards from descriptions of the standard changes with age in BMI is also thought to be difficult. Therefore, to obtain universal findings on physical growth phenomena, mathematical functions have often been applied. In this process Fujii (2006) advocated a wavelet interpolation method and attempted to analyze physical growth with it [11]. In this study, wavelet interpolation was applied to the changes in BMI with age in girls. In recent years, the very high risk of childhood obesity tracking to adulthood has been reported from the standpoint of preventive medicine. For people working in companies, avoiding obesity is a basic aspect of health management that decreases the risk of disease for the individual while also contributing to corporate productivity. However, since BMI shows an increasing trend in childhood, no evaluation method has been established. In this study, the changes with age in BMI were analyzed with a wavelet interpolation model, and evaluation chart that gives the standard curve for age-related change in BMI during childhood was constructed to identify obesity. Examinations were made of the validity of the obesity-thinness judgments and the physical development of the judged obesity types, and the tracking pattern of childhood obesity to adulthood was investigated from the standpoint of occupational health. The obesity tracking status was also investigated to explore the management of obesity risk in company.

\section{Methods}

\section{Subjects and Materials}

The subjects of this study were about 4,000 junior high school girls in the Tokai and western Japan regions. The birth dates of these third-year junior high school students were surveyed and school health records, as stipulated in the Law of School Health, were retrospectively examined. Longitudinal growth data on height and weight for girls from the first grade of elementary school (6-7 years old) to the third year of junior high school (14-15 years old) were obtained. Height and weight measurements were limited to those in April; other data were not used. Data on 350 obesity girls for whom all data were available were used in the analysis.

\section{BMI Calculations}

BMI is basically calculated by dividing weight $(\mathrm{kg})$ by the square of height $(\mathrm{m})$. In this study, a curve of the changes in BMI with age needed to be derived, and so BMI was calculated in each school year from the first grade of elementary school until the third year of junior high school to obtain longitudinal BMI data (Table 1). The mean and standard deviation for these longitudinal BMI values for each school year were calculated, and wavelet interpolation was applied to the mean changes in age with BMI.

\begin{tabular}{|c|c|c|l|c|l|l|l|l|l|}
\hline & \multicolumn{5}{|c|}{ Elementary school (N=4740) } & \multicolumn{3}{|l|}{ Junior high school (N=4740) } \\
\hline & 1 & 2 & 3 & 4 & 5 & 6 & 1 & 2 & 3 \\
\hline Mean & 15.97 & 16.24 & 16.72 & 17.17 & 17.69 & 18.44 & 19.42 & 20.18 & 20.79 \\
\hline SD & 2.02 & 2.19 & 2.49 & 2.70 & 2.83 & 2.98 & 3.14 & 3.07 & 3.04 \\
\hline \multicolumn{8}{c|}{ Table 1: Statistics of BMI during school age }
\end{tabular}

\section{Judgment of Obesity}

Five rank mean evaluation method was constructed by mean and standard deviation of BMI value based on age at nine grade. BMIs of all nine grade students were applied to the constructed mean evaluation chart. As the result, 349 obesity students were judged.

\section{Analysis Method}

The Wavelet Interpolation Model (WIM) is a method to examine growth distance values at adolescent peak and menarchal age. A growth curve is produced by data-data interpolation with a wavelet function and by deriving the growth velocity curve obtained by differentiating the described distance curve to approximately describe the true growth curve from given growth data. The effectiveness of the WIM lies in its extremely high approximation accuracy in sensitively reading local events. Details on theoretical background and the basis for this effectiveness are omitted here as they have already been set forth in prior studies by Fujii (1995) (1996)(1998)(1999)(2006) [11-15]. Wavelet interpolation was applied to the mean age distance values in BMI calculated from height and weight growth distance values from the first year of elementary school (6 years old) until the third year of junior high school (14 years old). The BMI age distance curve was then differentiated to identify the MPV age (age of maximum peak velocity in BMI during puberty) of BMI from the derived velocity curve. By constructing 5-step mean evaluation standard values for BMI values in each school year from the first grade of elementary school to the third year of high school with confirmation of the changes in the sigmoid shape of BMI, wavelet interpolation was applied to the obtained standard values. A BMI age evaluation chart was then constructed. 


\section{Analytical Procedures}

This describes the procedures for applying the wavelet interpolation model and deriving the results.

1) BMI was calculated from longitudinal height and weight records for first grade elementary school girls to third year junior high school girls, and the mean values and standard deviation for BMI in each year were calculated. The main age distance values and mean age velocity curve for the calculated BMI was drawn, and the MPV age of BMI was calculated from the drawn mean age velocity curve.

2) Wavelet interpolation was applied to the mean values and standard deviations of \pm 0.5 SD and \pm 1.5 SD for BMI in each school year, and an evaluation chart was constructed based on the changes with age in BMI.

3) Fat-lean levels were judged from the BMI age evaluation chart, and the rates of lean, moderate, and obese types were shown.

4) To investigate the normality of the BMI values in each school year, a normal distribution function for BMI values from age 6 to 14 was calculated, and the $\chi 2$ value was obtained.

\section{Normality of BMI in each School Year}

The $\chi^{2}$ values were derived from the below normal distribution function applied to BMI in each school year. The normal distribution function is shown below.

$$
f\left(x_{i}\right)=\frac{1}{\sqrt{2 \pi} \sigma} e^{-\frac{\left(x_{i}-\bar{X}\right)}{2 \sigma^{2}}}
$$

Looking at the $\chi 2$ values in each school year, statistical normality is seen in all school years. Significance $(\mathrm{P}<0.01)$ is also seen from tests of kurtosis and skewness (Table 2). These results suggest that it would be possible to construct an obesity evaluation chart from the standard age curve of BMI.

\begin{tabular}{|c|c|c|c|c|c|c|c|c|c|}
\hline & \multicolumn{6}{|c|}{ Elementary school } & \multicolumn{3}{c|}{ Junior high school } \\
\hline & 1 & 2 & 3 & 4 & 5 & 6 & 1 & 2 & 3 \\
\hline$\chi 2$ Value & 8282.1 & 6029.5 & 3472.1 & 2569.1 & 2473.9 & 2283 & 2674.4 & 3698.4 & 4063.2 \\
\hline
\end{tabular}

\section{Construction of an Evaluation Chart Derived from the Changes with Age in BMI}

Wavelet interpolation was applied to the mean BMI values from the first grade of elementary school to the third year of high school. Wavelet interpolation was then applied to the standard deviation values of the mean $\pm 0.5 \mathrm{SD}$ and $1.5 \mathrm{SD}$, and an evaluation chart was constructed based on the changes with age in BMI. The values for the evaluation segments for each age group are shown below.

Lean (-2 points) BMI $<-1.5 \sigma$

Somewhat lean $(-1$ point $)-1.5 \sigma<\mathrm{BMI}<-0.5 \sigma$

Average ( 0 points) $-0.5 \sigma<\mathrm{BMI}<0.5 \sigma$

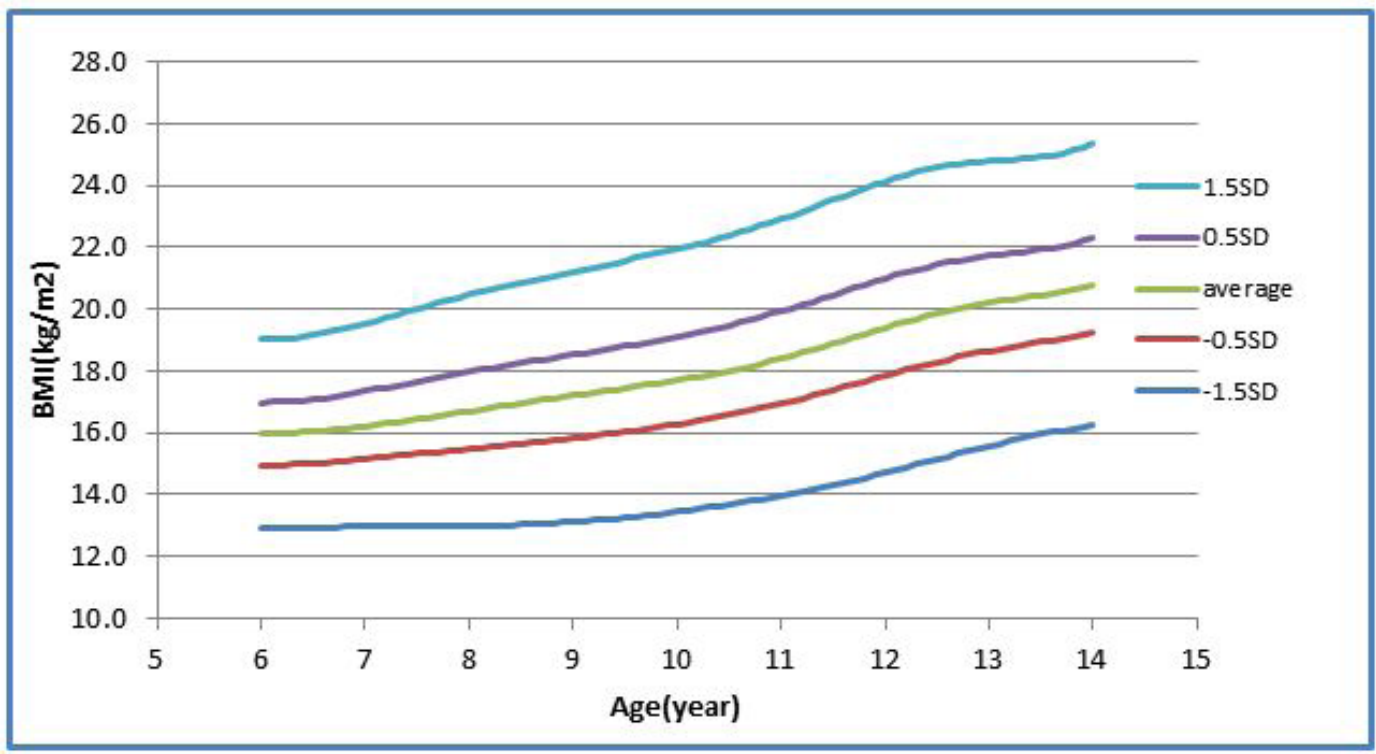

Figure 1: Fat-lean judgment chart based on change of BMI with age 
Somewhat obese (1 point) $0.5 \sigma<\mathrm{BMI}<1.5 \sigma$

Obese (2 points) $1.5 \sigma<\mathrm{BMI}$

The standard age curve for BMI is shown in Figure 1. This graph is an evaluation chart for the changes with age in BMI. As an example, when the BMI for the first year of junior high school is put in. In this way the evaluation chart for changes with age in BMI can be used to determine fat-lean level.

\section{Results}

A BMI age evaluation chart was applied to investigate how children determined to be obese at age 6 changed by the age of 14 . Figure 2,3, and 4 shows how children determined to be obese at age 6 tracked until the age of 14. Basically, the tracking index was taken to be the case when an individual stayed in the thin evaluation range steadily from the fifth grade of elementary school onward. When this index was applied, 256 of 349 children in the obese evaluation range from the fifth grade were judged to be obese at age 14. Among them, 106 were cases when the individuals were in the obese band in all grades. Of the remainder, 56 were judged to be somewhat obese at age 14, 26 shifted from average to obese, and 11 shifted from obese to somewhat thin. None of the children shifted from obese to thin.

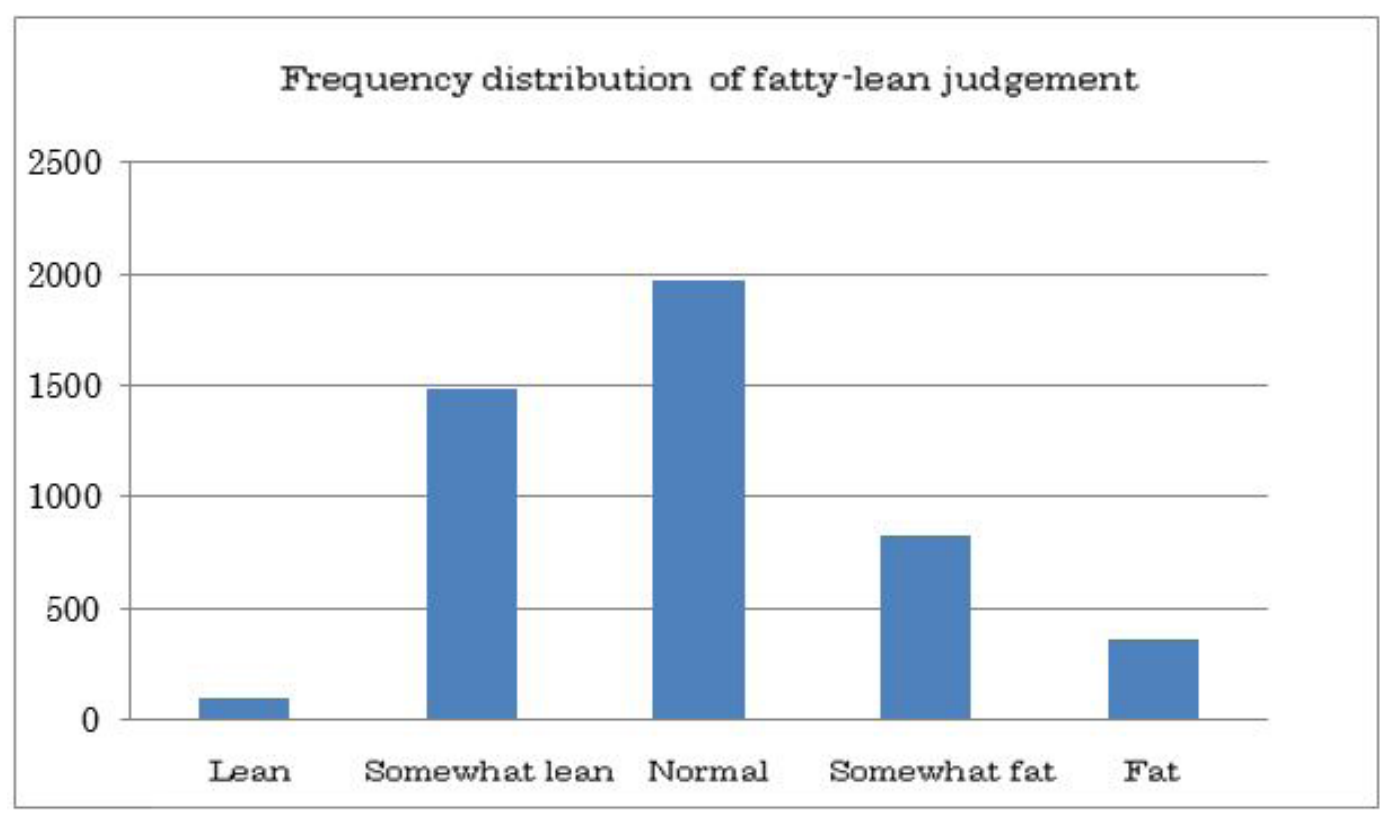

Figure 2: The frequency distribution judged by fat-lean judgment chart

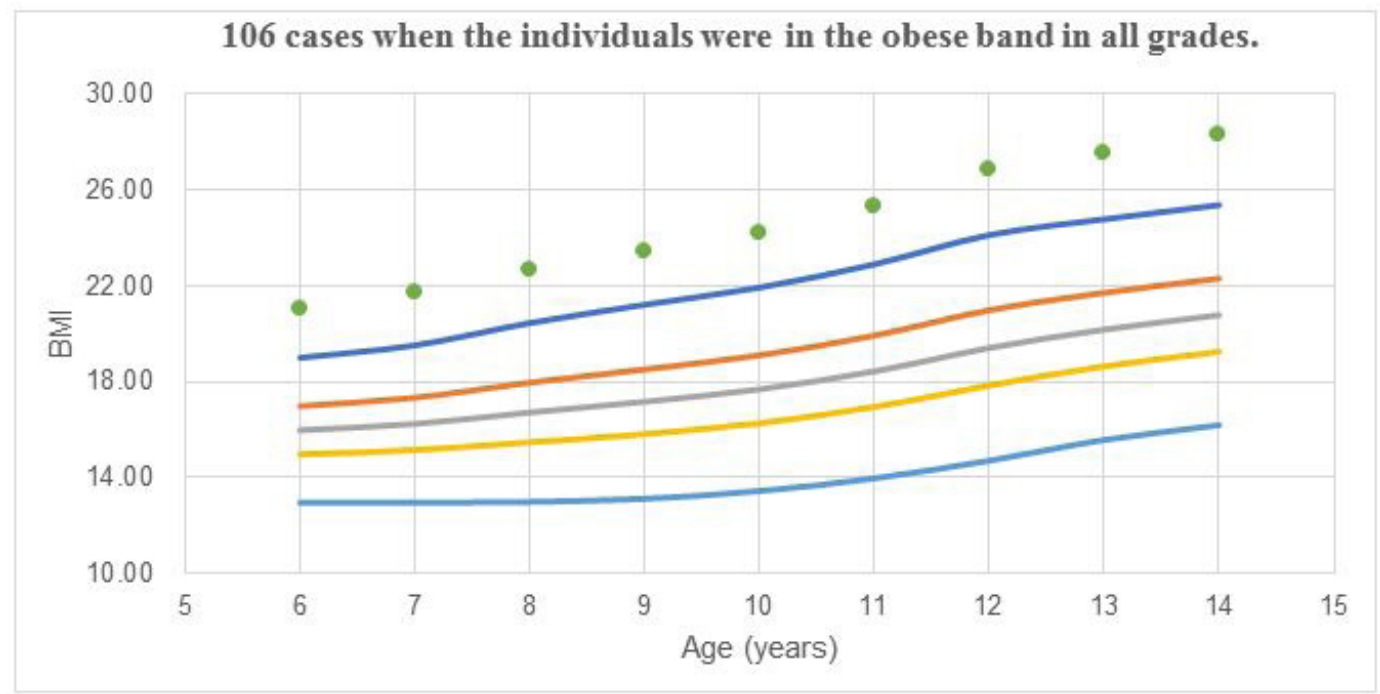

Figure 3: Tracking situation to have just been obesity 


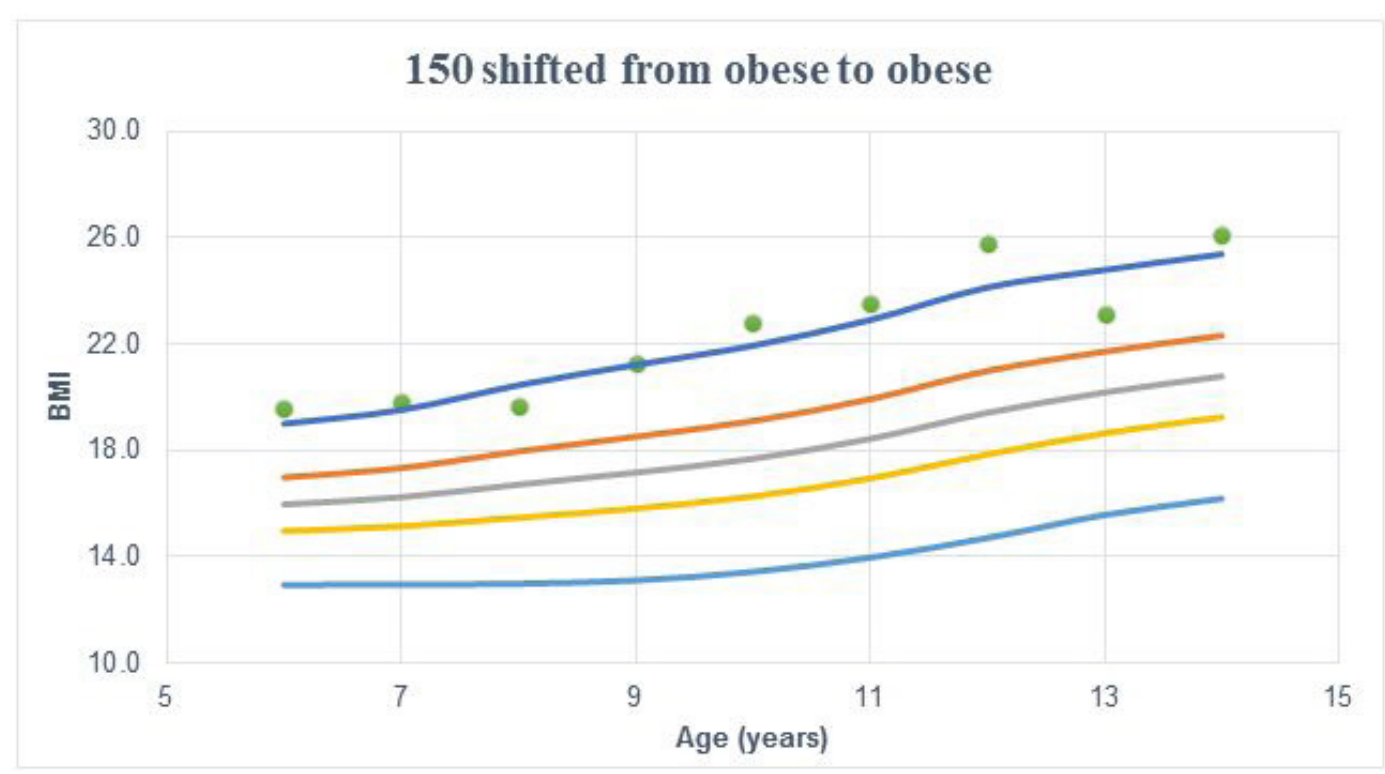

Figure 4: Tracking situation to have just been obesity and somewhat obesity

\section{Discussion}

Body mass index (BMI) is an index of physique created by Quetelet (1835), but even today it is not well understood what this index means [16]. It originated when Quetelet (1835) measured the physiques of 6,000 Scottish soldiers and 100,000 French enlisted men and derived the rule that the average adult weight is proportional to the square of height [16]. This became the Quetelet index. In fact, Kuroki (2007) has stated that BMI was first used not in the field of medicine but by the Japanese army prior to World War II. Of course, at that time the reports of Key et al.(1972) and Garrow and Webster (1985) had not yet appeared. BMI can be easily calculated, but there is no clear evidence for it as an index of body fat levels $[2,17,18]$. From forest ecology, Kuroki (2007) arrived at the fact that the ease with which a tree breaks and falls is related to its height and diameter, and said that when humans are also taken to be cylindrical one obtains the formula that has spread as the BMI calculation formula shown in the Introduction, and that this seems to be proportional to the thickness of the body [17]. When the correlation between abdominal girth and BMI from health check data was obtained without regard for differences in sex, it was found to be $r=0.9$. However, the detailed background for this is not clear and so unequivocal findings on the correlation between BMI and abdominal girth cannot be presented. The basis for the use of BMI by the pre-war Japanese army is not clear, but it certainly does not seem to be based on objective evidence. The use of BMI as an index expressing the level of obesity is based largely on the study of Key et al. (1972) [1]. Of course, since $\mathrm{BMI}$ is derived from values for height and weight, it obviously does not consider amount of body fat. Key et al. then demonstrated that BMI shows a high correlation with body fat percentage and level of obesity, and took it to be an important index in the field of body composition research. In addition to these findings, Teramoto et al. (1999), Otogi et al. (1999) investigated sex differences related to body fat distribution from the distribution of body composition in early childhood [19,20]. Garrows and Webster (1985) took the findings of Key et al. (1972) a further step forward and suggested that a meaningful index of body fat percentage is to be found in BMI [1,18]. In this study, however, the change in BMI with age was taken as a research question and no attempt was made to investigate the validity of the evaluation construct in determining fat-lean levels. In constructing an evaluation chart for fat-lean level that incorporates changes in BMI with age, there is an essential problem in calculating BMI. That is, while the value for BMI increases without limit, the minimum is 0 . The possibility that this contributes to the height of the obesity rate cannot be ruled out. The normality of the BMI values in each school year was then investigated, and normality was found. Thus, if the method to evaluate the mean value in each school year is found to be valid, evidence is obtained for the validity even when the evaluation band is constructed with wavelet interpolation applied to the mean and standard deviation. The results of application of the obese-lean evaluation chart showed a slight decreasing trend in the rate of appearance of the obese type. Since a period of sudden increase in height is detected in schoolgirls, an effect is thought to be shown on BMI calculations. Kori et al. (2013) warned about the risk of obesity progressing into adulthood, and the results of a comparison of the rate of change in BMI in the obese group and normal group suggest that a notable rate of change in the obese group heightens the risk for obesity in adulthood [21]. Although the rate of change in BMI in the obese group shows an unquestionably high value in the school years overall, with this finding alone the shift to adult obesity is not clear.

\section{Conclusion}

In this study the standard age change curve for BMI was described for the first time with the wavelet interpolation method using several thousand pieces of data, and an evaluation chart for levels of obesity was constructed from the first grade of elementary school to the third year of junior high school. This evaluation chart is useful in that a determination can be made in a very short 
time for people of any age. This may open a way forward for future examinations of the growth patterns of obese with the use of such longitudinal data. When the classification criteria for levels of obesity was slightly shifted, it was found that $90 \%$ of obese individuals retained the same type into adulthood when judging the obese type from the entire school-age period.

\section{References}

1. Arao T, Jinno H, Oida Y, Nagamatsu T (1998) The relational factor and life physical fitness of regional senior citizen. Japaneese J Public Health 45: 396-406.

2. Arao T, Jinno H, Oida Y, Kitabata Y, Egawa K, Mail Y (1999) Relation to the physical health condition and life physical fitness of regional senior citizen, Bulletin of The Physical Fitness Research Institute 96: 1-14.

3. Coakley EH, Kawachi I, Manson JE, Speirzer FE, Willet WC, et al. (1998) Lower levels of physical functioning are associated with higher body weight among middle-aged and older women. Int J Obes Relat Metab Disord 22: 958-65.

4. Davis JW, Cole TJ (1995) Body composition techniques in health and disease. Cambridge University press Symposium Series 3: 151-3.

5. Ferraro KF, Booth TL (1999) Age, body mass index, and functional illness. J Gerontol B Psychol Sci Soc Sci 54: 339-48.

6. Fujii K, Yamamoto Y (1995) The analysis of the growth velocity curve in height based upon the maturity rate. Japan J Physical Fitness Sports Medicine 44: 431-8.

7. Fujii K, Matsuura Y (1996) Analysis of velocity curve derived from mean growth curve in physique of boys. Japan J Physical Education Health Sport Sci 41: $247-60$.

8. Fuji K, Kawanami K (1998) An analysis in regard to relationship between age at MPV of height and weight, and its sex difference. Japanese J School Health 40: $317-31$.

9. Fujii K, Matsuura Y (1999) Analysis of the growth velocity curve for height by the Wavelet Interpolation Method in children classified by maturity rate. Am J Human Biol 11: 3-30.

10. Fujii K (2006) A Scientific Approach to Growth and Development-Physical Information Science for Growth and Development \& Health. Sankeisha, Nagoya.

11. Galanos AN, Pieper CF, Cornoni-Huntley JC, Bales CW, Fillenbaum GG (1994) Nutrition and function: is there a relationship between body mass index and the functional capabilities of community-dwelling elderly. J Am Geriatr Soc 42: 368-73.

12. Garrow JS, Webster J (1985) Quetelet's index (W/H2) as a measure of fatness. Int J Obes Relat Metab Disord 9: 147-53.

13. Keys A, Fidanza F, Karvonen MJ, Kimura N, Taylor HL (1972) Indices of relative weight and obesity. J Chronic Dis 25: 329-43.

14. Kori H, Sei M, Nakahori Y and Imoto I (2013) Impact of annual body mass index gain on obesity development in Japanese 6-year old non-obese children. Pediatr Int 55: 761-6.

15. Kuroki T (2007) Health, Aging, Longevity-Culture article of human and life-Chuohkoronshinshya, Tokyo, Japan.

16. Launer LJ, harris T, Rumpel C, Madans J (1994) Body mass index, weight change, and risk of mobility disability in middle-aged and older women. JAMA 271: 1093-8.

17. Lee M, Tanaka K, Nakadomo H, Watanabe K., Takeshima N, Hiyama T (1996) Effectiveness of physical fitness related to health for health evaluation index, J Exercise Sports Physioly 1: 79-89.

18. Otogi K, Komiya S, Teramoto K, Masuda T, Ube M (1999) Gender differences in body fat distribution of 3-to 6-year-old Japanese children. Jpn Phys Fitness Sports Med 48: 641-50.

19. Quetelet A (1835) Sur I' home et I' developpement de ses facultes. Essai sur physique sociale 2 Bachelier, Paris.

20. Rikli E, Jones CJ (1999) Development and validation of a functional fitness test for community-residing older adults. JAPA 7: 129-161.

21. Teramoto K, Otogi K, Komiya S (1999) Age-related changes body composition of 3-to 6-year-old Japanese Children. Appl Human Sci 18: 153-160.

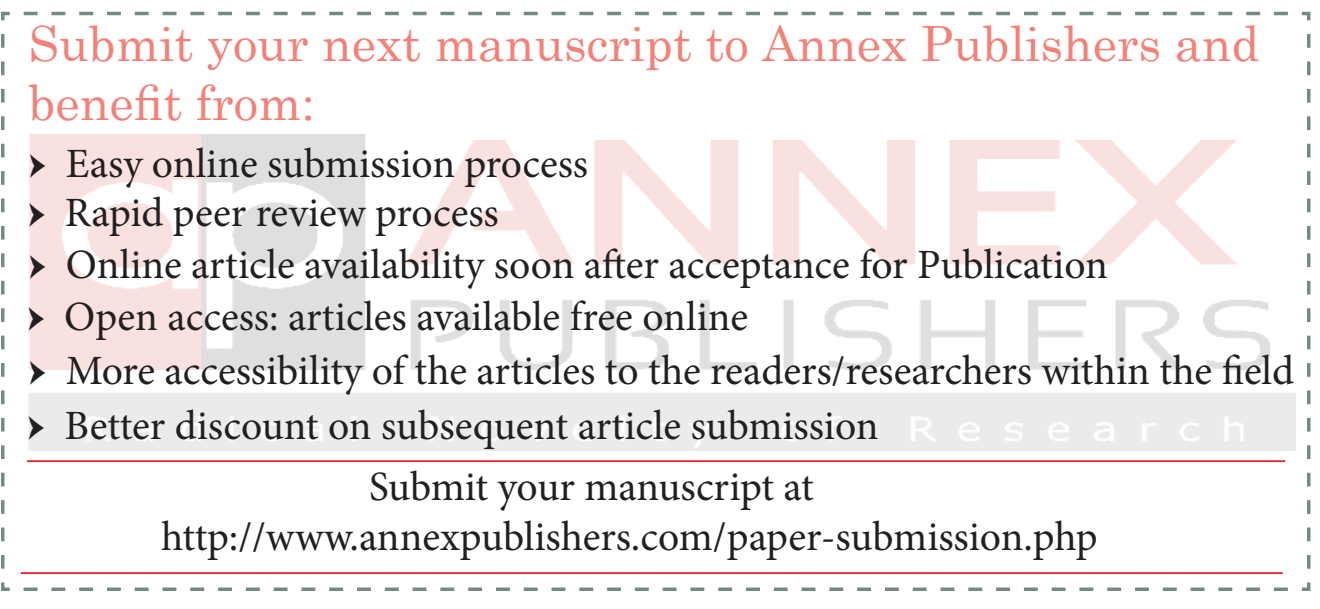

